CASE REPORT

Volume 15 Issue 12020

DOI: 10.21315/aos2020.15.1.410

ARTICLE INFO

Submitted: 17/09/2019

Accepted: 08/06/2020

Online: $15 / 07 / 2020$

\section{The Use of Customised Surgical Guide for Bilateral Inferior Mandibulectomy in a Case of Monostotic Fibrous Dysplasia}

\author{
Jun Ai Chong, Siti Salmiah Mohd Yunus*, Abd Jabar Nazimi, \\ Nordin Rifqah, Tan Huann Lan
}

Centre of Oral and Maxillofacial Surgery, Faculty of Dentistry, Universiti Kebangsaan Malaysia, 50300 Kuala Lumpur, Malaysia

"Corresponding author: sitisalmiah@ppukm.ukm.edu.my

To cite this article: Chong JA, Mohd Yunus SS, Nazimi AJ, Rifqah N, Lan TH (2020). The use of customised surgical guide for bilateral inferior mandibulectomy in a case of monostotic fibrous dysplasia. Arch Orofac Sci, 15(1): 73-80. https://doi.org/10.21315/aos2020.15.1.410

To link to this article: https://doi.org/10.21315/aos2020.15.1.410

\begin{abstract}
Fibrous dysplasia (FD) is a benign pathological condition of bone in which normal cancellous bone is replaced by immature woven bone and fibrous tissue. Surgical recontouring aims to remove the excess fibrous bone and achieve acceptable symmetry. Conventional surgery which depends on visual exposure and the surgeon's assessment during the procedure poses a great challenge in obtaining a predictable surgical outcome especially in bilateral presentation. Recent advances in surgical technology may overcome this challenge. Herein, we present a case of 23 years old Chinese lady who underwent bilateral inferior mandibulectomy for monostotic fibrous dysplasia. The aims of this surgery were to recontour the mandible symmetrically via extraoral approach while preserving the inferior alveolar nerves and mental nerves with the aid of a surgical guide. A stereolithography model of the patient's mandible was produced using the computed tomography (CT) scan data in Digital Imaging and Communications in Medicine (DICOM) format to assist in the surgery. Simulation with SurgiCase software (Materialise, Leuven, Belgium) was utilised in creating the customised surgical guide. The autoclavable surgical guide was manufactured by rapid prototyping technology and used intraoperatively to define the osteotomy line for mandibular recontouring. Virtual surgical planning greatly assists surgeons to deliver a good surgical result.
\end{abstract}

Keywords: Fibrous dysplasia; mandible; osteotomy; stereolithography; surgical guide

\section{INTRODUCTION}

Fibrous dysplasia (FD) is a rare benign developmental dysplastic disorder of which medullary bone is being replaced with cellular fibrous tissue containing foci of ossification (Nilesh et al., 2017). It was first documented by Lichtenstein in 1938 and accounts for $3 \%$ of all bony tumours. In $80 \%$ of cases, fibrous dysplasia affects a single bone (monostotic) (Nilesh et al., 2017). When multiple bones are involved (polyostotic), it can be associated with caféau-lait skin pigmentation and precocious puberty such as in McCune-Albright syndrome or present with intramuscular myxomas such as in Mazabraud syndrome (Wu et al., 2014). Bones affected by fibrous dysplasia can be surgically contoured for esthetic or functional purposes once the disease stabilizes and become quiescent, usually when skeletal maturity is attained (Jeyaraj and Srinivas, 2014). 
Virtual surgical planning and computeraided design (VSP-CAD) technology promotes new surgical techniques that assist in achieving an optimal anatomical and functional surgical result (Rohner et al., 2013). The preoperative surgical planning that involves surgical simulation of the resective phase together with the construction of stereolithographic models and surgical guides helps in improving precision and efficiency during surgery (Hegab, 2015). It minimises surgical time and helps anticipate intraoperative complications. A detailed history and clinical examination with precise imaging of the affected region is keystone in the preoperative surgical planning. The exact size and shape of the patient's craniofacial complex is attained through threedimensional (3D) imaging such as computed tomography (CT) scans (Efanov et al., 2018). Also, the fibrous dysplasia lesions can be mapped out through bone scintigraphy (Bousson et al., 2014).

This paper highlights the workflow of VSPCAD and the advantages of a customised surgical guide for bilateral inferior mandibulectomy in a case of monostotic fibrous dysplasia of the mandible.

\section{CASE PRESENTATION}

A 23-year-old Chinese lady was referred to the Oral Maxillofacial Department of Hospital Universiti Kebangsaan Malaysia for management of bilateral mandibular swelling. She presented with multiple episodes of mandibular pain since she was eight years old. Her mandible slowly increased in size over the years with no history of trauma to the mandible.

Extraorally (Fig. 1), she had diffused mandibular swelling with normal overlying skin colour and temperature. The bony swelling was hard in consistency and nontender. Her marginal mandibular branch was intact and there was no paraesthesia at her labiomental region bilaterally. There were no café au lait lesions on her body. Intraorally, there was buccal expansion of her mandible but no teeth displacement.

Her orthopantomogram (OPG) (Fig. 2) showed enlarged mandible with irregular moth-eaten appearance and scattered lytic areas within. Bone scintigraphy showed intense tracer accumulation only at the mandible indicating monostotic involvement. A high resolution multi slice $3 \mathrm{D}$ CT scan (Fig. 3) showed expanded mandible with thickened cortex and the bony matrix was predominantly sclerotic with scattered lytic areas, resembling irregular moth-eaten appearance as seen in her OPG. Irregular soft tissue density within the lytic lesions in the symphysis menti and both sides of the body of mandible was also noticeable.

An incisional biopsy under general anaesthesia was performed and the histopathological findings were consistent
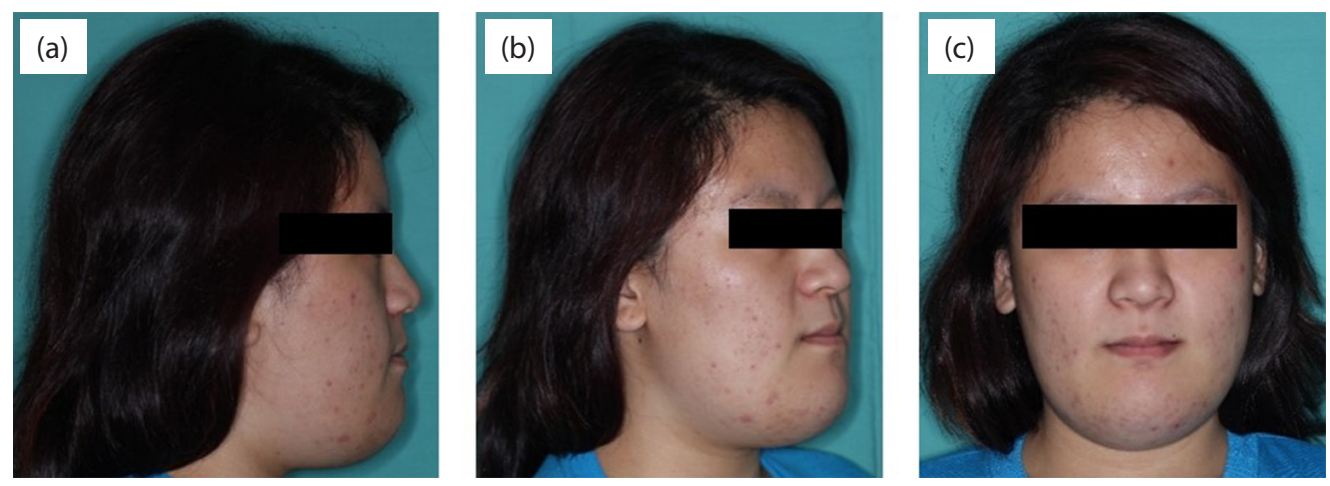

Fig. 1 Clinical photographs of mandibular enlargement: (a) Right lateral view (b) $45^{\circ}$ profile view (c) Frontal view. 


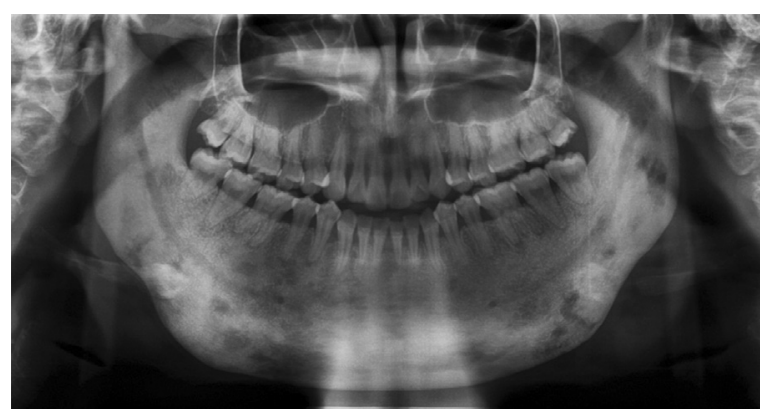

Fig. 2 Orthopantomogram of enlarged mandible.

with fibrous dysplasia. The surgery was planned with the aim of optimal tumour resection and symmetrical jaw appearance with preservation of vital structures, namely the teeth vitality, and both the inferior alveolar and mental nerves when the authors decided for the computerised planning.

The VSP-CAD workflow involved digital surgical planning, patient-specific guide design, surgical guide fabrication, and biomodel fabrication. The CT data in Digital Imaging and Communications in Medicine (DICOM) medical image file format was uploaded to the Surgicase CMF software (Materialise, Leuven, Belgium). The 3D virtual planning of the surgical guide was performed with consideration of the adjacent vital structures and prepared for an extraoral surgical approach. The amount of reduction and distance from the inferior alveolar nerves was set at $7.5 \mathrm{~mm}$ to $10.4 \mathrm{~mm}$ away from the vital structure bilaterally. The span of the surgical guide encompasses the lower border of the mandible with four anchor screws (Fig. 4).

The polyamide surgical guide was manufactured using selective laser sintering (SLS) through the SurgiCase CMF system (Materialise, Leuven, Belgium). This process uses a carbon dioxide laser beam to solidify autoclaveable polyamide powder to build up the model in layers, using a slice thickness of

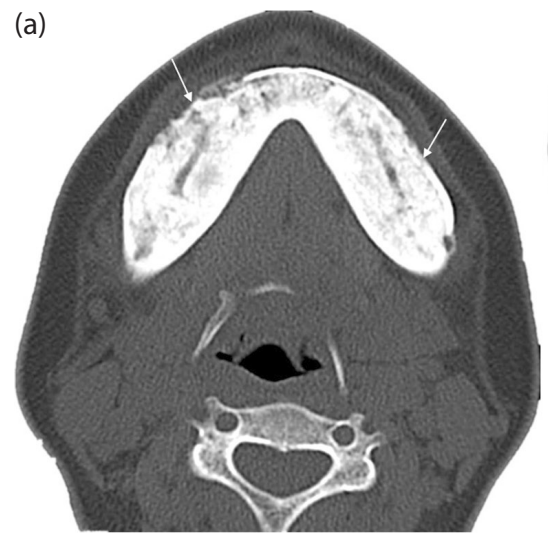

(b)

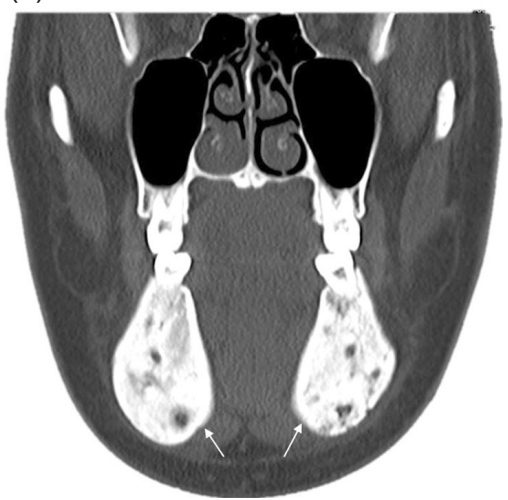

Fig. 3 (a) Axial slice of the CT scan showing thickened sclerotic cortex with scattered lytic areas and irregular soft tissue density (white arrows). (b) Coronal slice of the CT scan showing expanded mandible with thickened cortex.

(a)

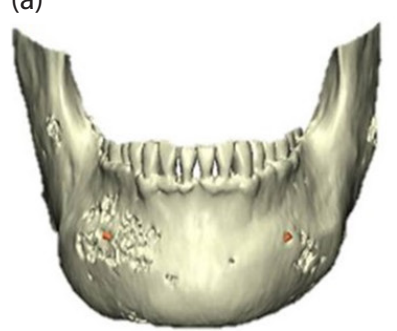

(b)

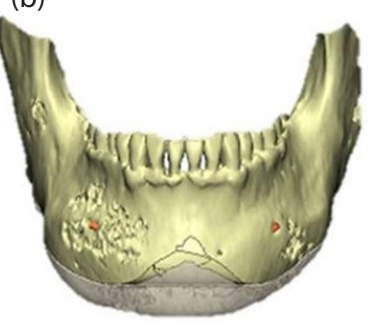

(c)

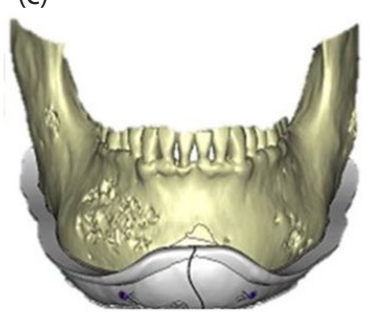

(d)

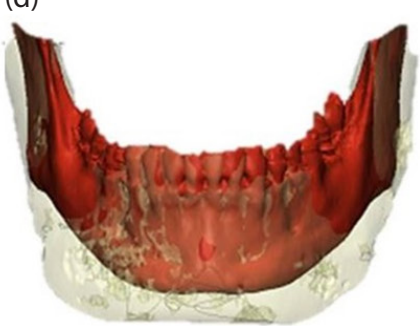

Fig. 4 3D virtual planning: (a) Initial situation (b) Planned resection (c) Surgical guide (d) Planned outcome. 
$0.1 \mathrm{~mm}$. The completed surgical guide was cleaned ultrasonically to remove all loose powder particles and sterilised using standard steam sterilisation (Oh, 2018). Similarly, a rapid prototype biomodel of her mandible was constructed to enhance communication with the patient and family members regarding the surgery.

The bilateral inferior mandibulectomy started with the usual scrubbing and draping via aseptic technique under general anaesthesia. The lower border of the mandible was exposed via visor incision (Fig. 5). The incision was done from right to left angle of mandible, by placing the incision $2 \mathrm{~cm}$ away from the lower border of the mandible. Incision started through the skin and to the subcutaneous layers. Then blunt dissection was performed through the subplatysma layer and the deep cervical fascia was identified. Subsequently the right and left facial vein were identified and ligated. Lower border of the mandible was identified and incision through the periosteum was done to expose the lower border of the mandible. The customised surgical guide was placed and fixed with four anchor screws [Synthes osteosynthesis titanium plating system-matrix mandible screw: 3 screws of $2.0 \mathrm{~mm}$ (diameter) $\times$ $7.0 \mathrm{~mm}$ (length); 1 screw of 2.0 (diameter) $\times 12.0 \mathrm{~mm}$ (length)]. Bicortical osteotomy was carried out with oscillating saw that was placed perpendicular to the superior most aspect of the surgical guide (Fig. 6).

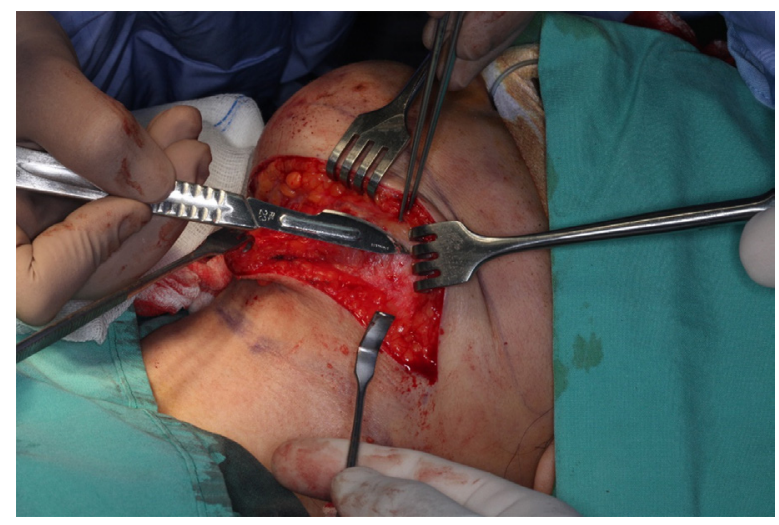

Fig. 5 Dissection to periosteum exposing mandible.
The chisel and mallet were also used to complete the osteotomy site whenever the maximal depth of the saw was reached. Finally, the edges of the inferior border of mandible were smoothened prior to its closure (Fig. 7). Bilateral Redivac drains placed bilaterally and layer closure was carried out with $3 / 0$ absorbable sutures.

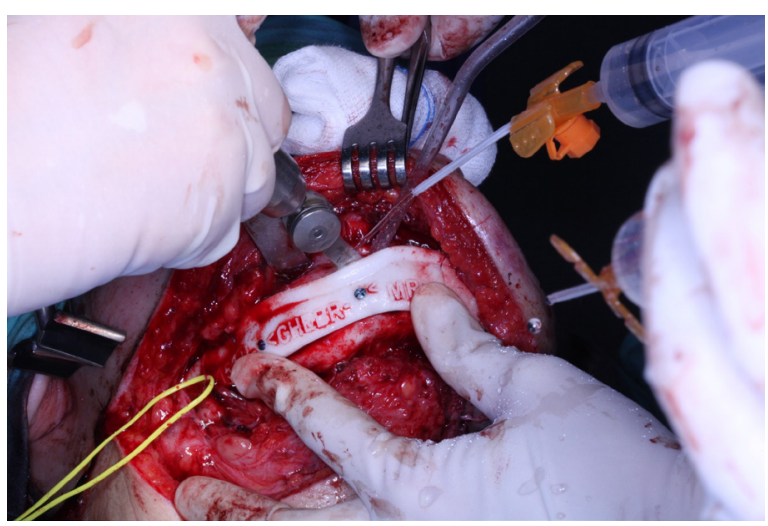

Fig. 6 Bone cut with oscillating saw and irrigation with the customised surgical guide in place.

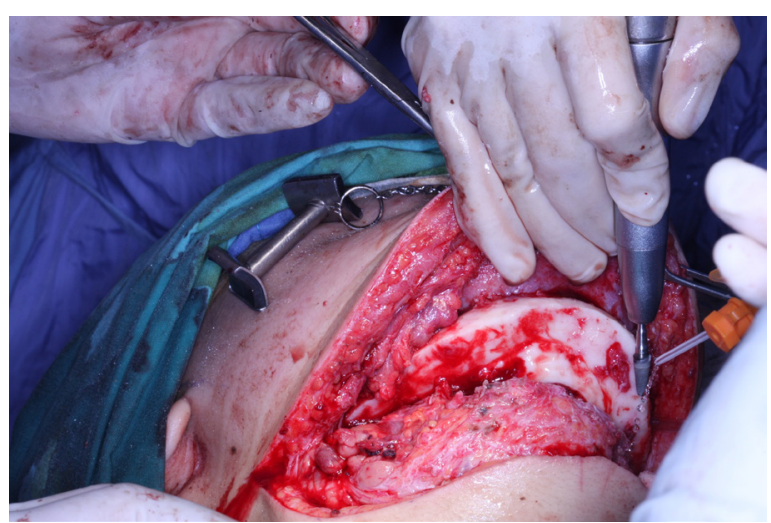

Fig. 7 Smoothening the edges of the inferior border of mandible.

Postoperative assessment showed intact marginal mandibular branch of the facial nerve. There was also no paraesthesia of the labiomental region. Symmetrical reduction of the mandible was achieved with good healing. Postoperative CT scan taken at three months later showed considerable amount of bony osteotomy achieved (Fig. 8). Clinical photographs taken at four years review showed no obvious mandibular enlargement and good scar healing (Fig. 9). 


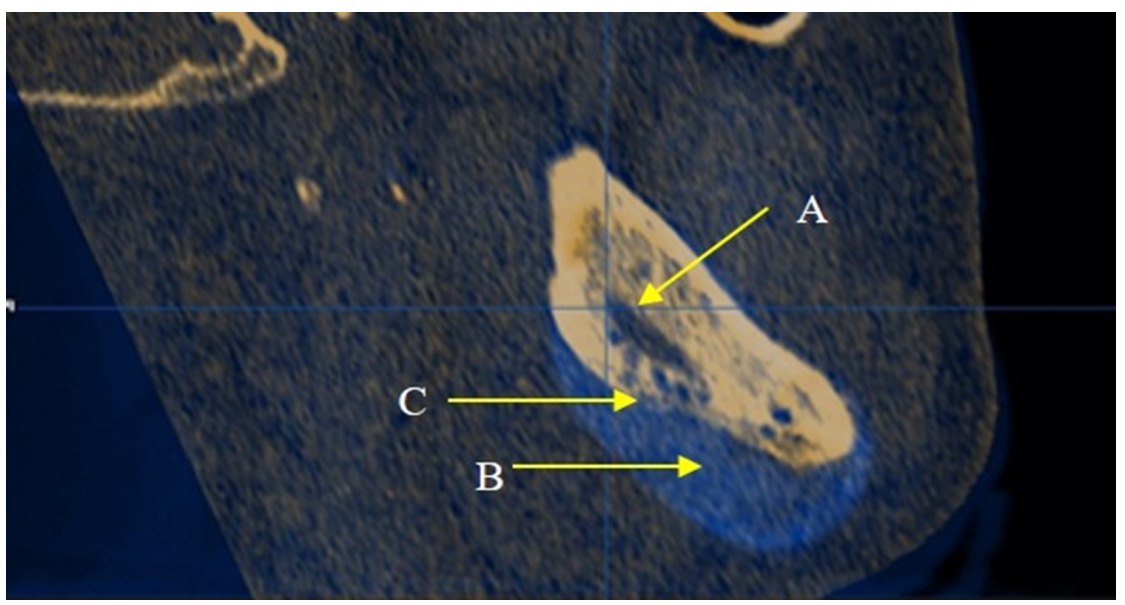

Fig. 8 Image fusion of right body of mandible showing bone reduction with inferior alveolar nerve preservation. Arrow A shows inferior alveolar nerve canal, arrow B shows the amount of bone reduction and arrow $C$ shows the inferior border of mandible.
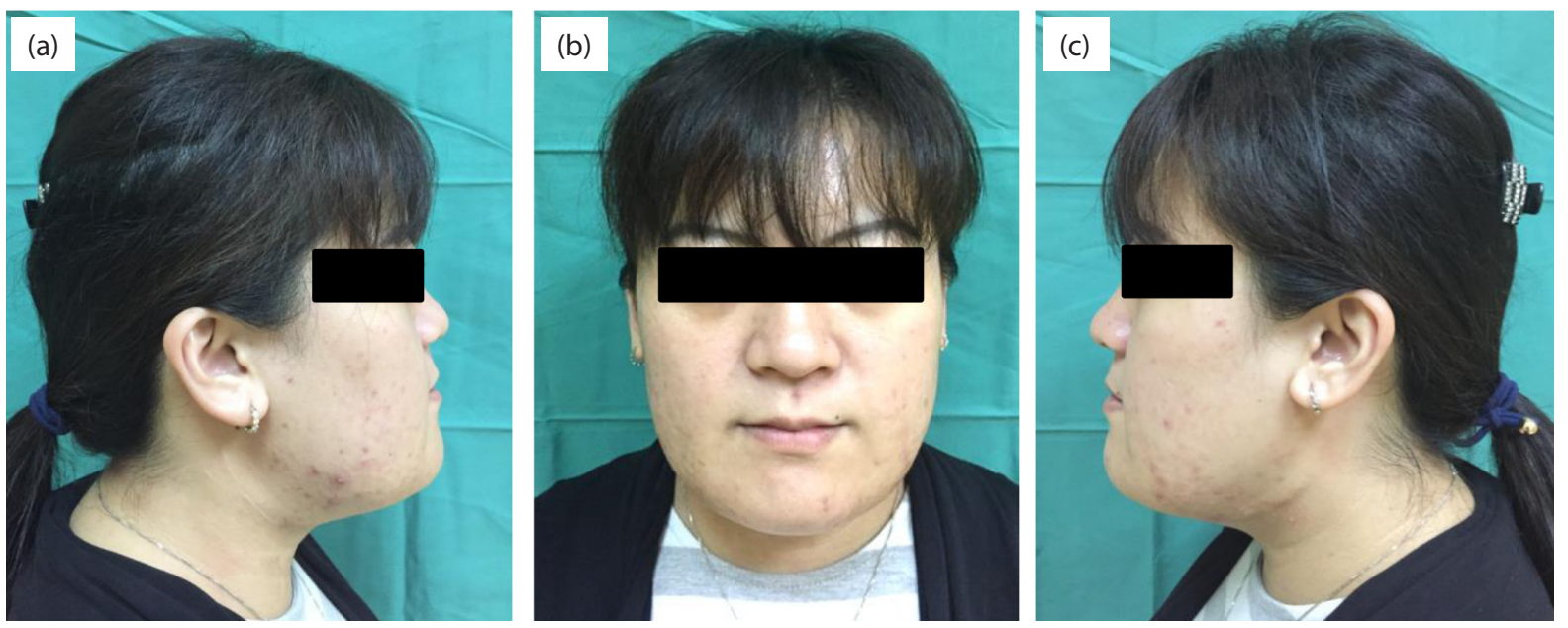

Fig. 9 Clinical photographs at four years review: (a) Right lateral view (b) Frontal view (c) Left lateral view.

\section{DISCUSSION}

In this case report, we demonstrated virtual surgical planning in fabrication of a customised surgical guide and biomodel in a case of monostotic fibrous dysplasia of the mandible. Production of geometrically complex shaped models have long been made possible with rapid prototyping and currently used in orthognathic and craniomaxillofacial surgery, traumatology and dental implantology surgeries (Oh, 2018). The biomodels improves communication between doctors and patients and aids in informed consent and can be used in simulating the surgery prior to the actual surgery (Matias and Zenha, 2017). On the other hand, the use of customised printed surgical guide has also been described (Hoang et al., 2016). Wide material selections have allowed for good stiffness and wear resistance of the surgical guide. This enables the surgical guide to maintain its shape accurately without inadvertent damage during intraoperative handling. The need for 3D virtual planning with a customised surgical guide is of importance in this case because there was no normal reference mirror available for surgical recontouring of the mandible. Intraoperative assessment may lead to poor surgical outcome and lengthening of the surgical duration. 
The amount of reduction was set based on generic mandible data of 'normalised' lower border of mandible contour from the software provider (Materialise, Leuven, Belgium). On hindsight, the data gathered on the mean shapes of the outer mandible contour in producing standardized preformed reconstruction plates could be used in our case (Metzger et al., 2011). The other aspect that could be improved is to calculate on the soft tissue response following the osteotomy. However, soft tissue response is only partially predictable depending on individual's morphology, thickness and elasticity of the tissue (Wermker et al., 2014). No reduction of the lingual aspect of the mandible was performed because it does not contribute to a better aesthetics outcome. This decision was also made as to prevent unnecessary complications such as airway compromise, avoiding sublingual bleed and disruption of muscle attachments. The muscle attachments could be in an abnormal position that could not be well visualised within the virtual planning software used. Unnecessary disruption to these groups of muscles may lead to some other complications such as mouth opening and deglutition.

Although an intraoral approach is more suitable for surgical recontouring to achieve better aesthetics of the mandible, there would be difficulty in placing the surgical guide directly onto the mandible due to the presence of the mental nerve. Hence, the surgical guide can be trimmed down at the area of the mental nerve if an intraoral approach is used but this may affect the stabilisation of the surgical guide (Kim et al., 2015). Additionally, the mental foramens bilaterally were narrowed down by the lesion in this case. Any vast intraoral access may increase risks of injury and defeat the purpose of the virtually-designed surgical plate. Also, osteotomy peripheral to the mental foramen will also risk further injury to the nerve as the periosteal layer that cannot be well-defined secondary to multiple cortical perforations and tethering. Therefore, based on these factors, we performed the surgical recontouring via extraoral approach.

The surgical guide should be stable, well fitting, easy to fix, and not interfering with the surgical site (Ren et al., 2018). Interlocking system between two guide parts to ease the placement of the surgical guide should be considered during the surgical guide design. This allows handling of the guide as two different units with midline interlock to retain its position and stability. The surgical guide can also be slightly extended further to the lingual side to increase its stability by allowing it to entirely 'wrap' around the deformity. However, this is only possible when the lesion is approached extraorally. Cutting slots can also be incorporated for more accurate placement of the saw blade. Finally, additional fixation holes (more than four) can be prepared and additional emergency screws should be made available in the surgical kit in case of loose fixation screws that may affect its stability.

There is high level of precision in the execution of mandibular osteotomies with surgical cutting guides (Rodby et al., 2014). It was also suggested that the surgical accuracy and safety can be further improved by using navigation system for more predictability and effectiveness (Gui et al., 2013). Although most lesions stop growing after skeletal maturation, hormonal changes from pregnancy or use of oral contraceptives may stimulate growth or result in the development of lesions within the area of fibrous dysplasia such as aneurysmal bone cysts. Therefore, the patient should have long term monitoring. Thus, the post-operative CT scan is important for future comparisons (Regu et al., 2014). Both reduction and even if there is a re-growth, the dimension could be quantified throughout the series of CT scans. Most importantly, the clinical outcome is acceptable to the patient especially in consideration of no damage to the adjacent nerves or teeth. 


\section{CONCLUSION}

Virtual surgical planning greatly assists surgeons to deliver a good surgical result and prevent surgical complications to the adjacent vital structures. Therefore, implementation of this technology in elective surgeries should be considered.

\section{ACKNOWLEDGEMENTS}

We wish to thank the patient for giving us permission to present these findings and all the people who were involved with the management of this patient.

\section{REFERENCES}

Bousson V, Rey-Jouvin C, Laredo JD, Le Merrer M, Martin-Duverneuil N, Feydy A et al. (2014). Fibrous dysplasia and McCuneAlbright syndrome: Imaging for positive and differential diagnoses, prognosis, and follow-up guidelines. Eur $\mathcal{F}$ Radiol, 83(10): 1828-1842. https://doi.org/10.1016/j.ejrad .2014 .06 .012

Efanov JI, Roy AA, Huang KN, Borsuk DE (2018). Virtual surgical planning: The pearls and pitfalls. Plast Reconstr Surg Glob Open, 6(1): e1443. https://doi.org/10.1097/ GOX.0000000000001443

Gui H, Zhang S, Shen SG, Wang X, Bautista JS, Voss PJ (2013). Real-time imageguided recontouring in the management of craniofacial fibrous dysplasia. Oral Surg Oral Med Oral Pathol Oral Radiol, 116(6): 680-685. https://doi.org/10.1016/ j.00oo.2013.07.012

Hegab A (2015). The cutting edge in oral and maxillofacial surgery. F Oral Hyg Health, 3: 184. https://doi.org/10.4172/2332-0702 .1000184
Hoang D, Perrault D, Stevanovic M, Ghiassi A (2016). Surgical applications of threedimensional printing: A review of the current literature and how to get started. Ann Transl Med, 4(23): 456. https://doi. org/10.21037/atm.2016.12.18

Jeyaraj CP, Srinivas CV (2014). Craniofacial and monostotic variants of fibrous dysplasia affecting the maxillofacial region. F Oral Maxillofac Surg Med Pathol, 26(3): 424-431. https://doi.org/10.1016/ j.ajoms.2013.05.011

Kim H, Kim NK, Lim JH, Kim HJ (2015). Surgical guide for symmetrical aesthetic surgery in unilateral fibrous dysplasia. Br f Oral Maxillofac Surg, 53(7): 671-673. https://doi.org/10.1016/j.bjoms .2015 .04 .012

Matias M, Zenha H (2017). Three-dimensional printing: Custom-made implants for craniomaxillofacial reconstructive surgery. Craniomaxillofac Trauma Reconstr, 10(2): 89-98. https://doi.org/10 $.1055 / \mathrm{s}-0036-1594277$

Metzger MC, Vogel M, Hohlweg-Majert B, Mast H, Fan X, Rüdell A et al. (2011). Anatomical shape analysis of the mandible in Caucasian and Chinese for the production of preformed mandible reconstruction plates. $\mathcal{F}$ Craniomaxillofac Surg, 39(6): 393-400. https://doi.org/10 $.1016 /$ j.jcms.2010.10.005

Nilesh K, Punde P, Parkar MI (2017). Craniofacial fibrous dysplasia of zygomaticomaxillary complex. World $\mathcal{F}$ Plast Surg, 6(1): 369-374.

Oh JH (2018). Recent advances in the reconstruction of cranio-maxillofacial defects using computer-aided design/ computer-aided manufacturing. Maxillofac Plast Reconstr Surg, 40(1): 2. https://doi.org/ 10.1186/s40902-018-0141-9 
Regu P, Jayachandran S, Meenapriya PK (2014). A case of isolated craniofacial fibrous dysplasia - Radiologist's perspective. I Pierre Fauchard Acad, 28(1): 5-10. https://doi.org/10.1016/j.jpfa.2014.03.001

Ren W, Gao L, Li S, Chen C, Li F, Wang Q et al. (2018). Virtual planning and 3D printing modeling for mandibular reconstruction with fibula free flap. Med Oral Patol Oral Cir Bucal, 23(3): e359-e366. https://doi .org/10.4317/medoral.22295

Rodby KA, Turin S, Jacobs RJ, Cruz JF, Hassid VJ, Kolokythas A et al. (2014). Advances in oncologic head and neck reconstruction: Systematic review and future considerations of virtual surgical planning and computer aided design/computer aided modeling. F Plast Reconstr Aesthet Surg, 67(9): 1171-1185. https://doi.org/10.1016/j.bjps .2014 .04 .038
Rohner D, Guijarro-Martínez R, Bucher P, Hammer B (2013). Importance of patient-specific intraoperative guides in complex maxillofacial reconstruction. $\mathcal{f}$ Craniomaxillofac Surg, 41(5): 382-390. https://doi.org/10.1016/j.jcms.2012.10.021

Wermker K, Kleinheinz J, Jung S, Dirksen D (2014). Soft tissue response and facial symmetry after orthognathic surgery. $\mathcal{F}$ Craniomaxillofac Surg, 42(6): e339-e345. https://doi.org/10.1016/j.jcms.2014.01.032

Wu H, Yang L, Li S, Jin X, Xu J, Lu J et al. (2014). Clinical characteristics of craniomaxillofacial fibrous dysplasia. $\mathcal{F}$ Craniomaxillofac Surg, 42(7): 1450-1455. https://doi.org/10.1016/j.jcms.2014.04.009 